\title{
Exploring new mechanisms for effective antimicrobial materials: Electric Contact-killing based on multiple Schottky barriers
}

\author{
Eva de Lucas-Gil ${ }^{1 *}$, Julián J. Reinosa ${ }^{1}$, Kerstin Neuhaus ${ }^{2}$, Liliana Vera ${ }^{3}$, Marisol Martín- \\ González ${ }^{3}$, José F. Fernández $z^{1}$ and Fernando Rubio-Marcos ${ }^{1}$
}

${ }^{1}$ Electroceramic Department, Instituto de Cerámica y Vidrio, CSIC, Kelsen 5, 28049 Madrid, Spain

${ }^{2}$ University of Münster, Institute for Inorganic and Analytical Chemistry, Corrensstr. 28/30, D-48149 Münster, Germany ${ }^{3}$ IMM - Instituto de Microelectrónica de Madrid (CNM-CSIC), Isaac Newton 8, PTM, E-28760, Tres Cantos, Spain

*Correspondence and requests for materials should be addressed to E. L-G. (email: elucas@icv.csic.es)

\begin{abstract}
The increasing threat of multidrug-resistance organisms is a cause for worldwide concern. Progressively microorganisms become resistant to commonly used antibiotics, which are a healthcare challenge. Thus, the discovery of new antimicrobial agents or new mechanisms different from those used is necessary. Here, we report an effective and selective antimicrobial activity of microstructured $\mathrm{ZnO}(\mathrm{Ms}-\mathrm{ZnO})$ agent through the design of a novel star-shaped morphology, resulting in modulation of surface charge orientation. Specifically, we find that Ms-ZnO particles are composed of platelet stacked structure, which generates multiple Schottky barriers due to the misalignment of crystallographic orientations. We also demonstrated that this effect allows to the negative charge accumulation in localized regions of the structure acting as "charged domain walls", thereby improving the antimicrobial effectiveness by electric discharging effect. We use a combination of field emission scanning electron microscopy (FE-SEM), SEM-cathodoluminescence imaging and Kelvin probe force microscopy (KPFM) to determine that the antimicrobial activity is a result of microbial membrane physical damage caused by direct contact with the Ms-ZnO agent. It is important to point out that $\mathrm{Ms}-\mathrm{ZnO}$ does not use the photocatalysis or the $\mathrm{Zn}^{2+}$ released as main antimicrobial mechanism, so consequently this material would show low toxicity and robust stability. This approach opens new possibilities to understand both the physical interactions role as main antimicrobial mechanisms and insight into the coupled role of hierarchical morphologies and surface functionality on the antimicrobial activity.
\end{abstract}

KEYWORDS: $\mathrm{ZnO}$, microstructure, antimicrobial activity, action mechanism, physical interactions, surface charge orientation 
E. de Lucas-Gil, J.J. Reinosa, K. Neuhaus, L. Vera-Londono, M. Martín-González, J.F. Fernández, F. Rubio-Marcos, Exploring New Mechanisms for Effective Antimicrobial Materials: Electric Contact-Killing Based on Multiple Schottky Barriers, ACS Appl. Mater. Interfaces. 9 (2017) $26219-26225$. doi:10.1021/acsami.7b09695

\section{Introduction}

Recently, the society is becoming aware of the increasing threat of multidrug-resistant organisms (MDROs). The MDROs represent a significant worldwide health risk and pose a serious healthcare challenge. The impact is so serious that the World Health Organization has recently drawn up a list of resistant bacteria to guide research, discovery, and development of new antibiotics. ${ }^{1}$ The overuse of antibiotics, inappropriate antibiotic use in selecting resistant organisms and the ability of resistant organisms to spread in the hospital setting are the main causes of antimicrobial resistance. ${ }^{2}$ These malpractices foster the evolution of antibiotic resistant strains. At present, more than 890 such unique enzymes have been discovered far more than the antibiotics developed to combat them..$^{2,3}$ Therefore, novel antibacterial strategies are critical to address the drug resistance and tolerance of antibiotics against pathogens. ${ }^{4-6}$ Generally, the most used agents are organic. However, inorganic antimicrobial agents have higher stability than organic materials. ${ }^{7,8}$ Thus, a new route could consist of a change of antimicrobial agents and/or action mechanisms. One of the inorganic materials with antimicrobial properties is $\mathrm{ZnO}$. Much of the literature in this field has focused on antibacterial applications. ${ }^{9-11}$ Despite the vast literature on the antimicrobial properties of $\mathrm{ZnO}$, only a handful of reports have investigated the antifungal activity. 12,13 Analyzing in detail the mechanisms by which the $\mathrm{ZnO}$ antimicrobial agent is effective we find three kinds: (i) chemical interactions, (ii) physical interactions and (iii) a combination of the physical and chemical interaction. ${ }^{14}$ Within the chemical mechanisms include $\mathrm{Zn}^{2+}$ cation release and reactive oxygen species (ROS) generation that react with the cell envelope components. On the other hand, abrasion, penetration and direct interaction through electrostatic effect are found in physical mechanisms. The most studies of the $\mathrm{ZnO}$ antimicrobial mechanism refer to ROS generation as the main mechanism.7,14-17 In addition, other authors report the importance of $\mathrm{Zn}^{2+}$ release ${ }^{18}$ and the combination between ROS and $\mathrm{Zn}^{2+}$ to get the antimicrobial property. ${ }^{19}$ Even the combination of physical and chemical methods to combat microorganisms is mentioned in the literature. ${ }^{20-22} \mathrm{~A}$ disadvantage of these mechanisms based primarily on chemical interactions is the potential toxicity of cation released or radicals generated i.e. ROS production has been associated with the development of many diseases such as cancer, atherosclerosis, diabetes, and neurodegeneration. ${ }^{17}$ Hence, a need exists for antimicrobial agents which improve the MDROs mortality and avoid the toxic side effects they generate. 
E. de Lucas-Gil, J.J. Reinosa, K. Neuhaus, L. Vera-Londono, M. Martín-González, J.F. Fernández, F. Rubio-Marcos, Exploring New Mechanisms for Effective Antimicrobial Materials: Electric Contact-Killing Based on Multiple Schottky Barriers, ACS Appl. Mater. Interfaces. 9 (2017) $26219-26225$. doi:10.1021/acsami.7b09695

For the present study, we provide a multidomain $\mathrm{ZnO}$ microparticles having a star-shaped morphology as antimicrobial key in the development of physical interactions to combat MDROs. For that, different tests are realized by using multidrug-resistance microorganism, both bacteria and fungi. At the same time, the antimicrobial mechanism of such microstructured $\mathrm{ZnO}$ has been meticulously studied. These results confirm the low chemical activity respect to physical interactions against microorganisms. Accordingly, the main role of physical mechanism makes the microstructured $\mathrm{ZnO}$ a novel antimicrobial agent with methods not used until now against the fight of MDROs.

\section{Results and Discussion}

Characterization of microstructured $\mathrm{ZnO}$. The synthesis of $\mathrm{ZnO}$ (thereafter microstructured $\mathrm{ZnO}, \mathrm{Ms}-\mathrm{ZnO}$ ) by soft chemistry method starts with urea $\left(\mathrm{CO}\left(\mathrm{NH}_{2}\right)_{2}\right)$ and zinc nitrate $\left(\mathrm{Zn}\left(\mathrm{NO}_{3}\right)_{2} \cdot 6 \mathrm{H}_{2} \mathrm{O}\right)$ solutions heated in an oil bath at $100-120^{\circ} \mathrm{C}$, see Experimental Section. At the end, the as-prepared product is thermally treated at $500^{\circ} \mathrm{C}$ for a short time, $5 \mathrm{~min}$, in the air. The obtained product $(\mathrm{Ms}-\mathrm{ZnO})$ is structurally characterized by X-ray diffraction (XRD). The XRD diffractogram of $\mathrm{Ms}-\mathrm{ZnO}$ is represented in Fig. S1. As shown, the Ms-ZnO diffraction pattern match to hexagonal wurtzite structure ZnO (JCPDS Card No. 36-1451). Likewise, following the behaviour of wurtzite, this structure grows along the c-axis, i.e. along [0001] crystallographic direction. No others peaks are identified so there are not reactant or secondary phases and only the typical crystalline structure of $\mathrm{ZnO}$ are presented. In addition, a morphological characterization is also studied by field emission scanning electron microscopy (FE-SEM). Low-resolution (LR) micrographs (Fig. 1a-b) show the well-defined star morphology of Ms-ZnO. The star-like particles are not agglomerated so they have a high capacity to be dispersed. The particle size is about ca. $1.5 \mu \mathrm{m}$ of diameter which establishes $\mathrm{Ms}-\mathrm{ZnO}$ at the microscale. Highresolution (HR) image (Fig. 1c) displays that star-like particles are formed by about 5 or 6 "branches" joined by a "central branch". At higher magnifications (Fig. 1d) of a branch, the micrograph shows that it is composed of platelets. These platelets are organized in stacks. To better define platelets characterization, a statistical study of the main morphological parameters is performed. The platelet diameter is represented in Fig. 1e. As shown, the platelet diameter manifests mostly a monomodal behaviour in the range of 100 to $500 \mathrm{~nm}$. There is an exception of two populations that are located at 350 and $450 \mathrm{~nm}$ which would fit the base platelets of the stacks. On the whole, the diameter average value is $\sim 200 \mathrm{~nm}$ that places the platelets outside the nanoscale (above $100 \mathrm{~nm}$ ). 
E. de Lucas-Gil, J.J. Reinosa, K. Neuhaus, L. Vera-Londono, M. Martín-González, J.F. Fernández, F. Rubio-Marcos, Exploring New Mechanisms for Effective Antimicrobial Materials: Electric Contact-Killing Based on Multiple Schottky Barriers, ACS Appl. Mater. Interfaces. 9 (2017) $26219-26225$. doi:10.1021/acsami.7b09695.

Other parameter studied is the platelet thickness or edge length (Fig. 1f). Again, a monomodal behaviour is observed where the average value of edge length corresponds to $76 \mathrm{~nm}$. Surprisingly, the platelets are not perfectly aligned. As shown in Fig. 1d, there is a rotation angle $(\theta)$ between the stacked platelets. The rotation angle $(\theta)$ between two adjacent platelets is calculated from the angle formed by two maximal radii (radius of the circumscribed circle which goes from the platelet center to one vertex) of adjacent platelets (see Fig. S2). The rotation angle $(\theta)$ occurs along the c-axis of the $\mathrm{ZnO}$ platelets. The Fig. $1 \mathrm{~g}$ represents the rotation angle histogram of platelets where an angle between $>0^{\circ}$ and $20^{\circ}$ prevails, with the exception of two small populations at higher angles $\left(30^{\circ}\right.$ and $\left.35^{\circ}\right)$. The average rotation angle of the platelets is ca. $13^{\circ}$. Therefore, Ms- $\mathrm{ZnO}$ is a micrometric structure built from small stacked platelets hierarchically organized (that is, a microstructure of platelets). Before checking the antimicrobial activity of $\mathrm{Ms}-\mathrm{ZnO}$, two $\mathrm{ZnO}$ samples with different sizes are used for comparative purposes: micrometric $\mathrm{ZnO}$ (referred as microZnO) and nanometric $\mathrm{ZnO}$ (called as nanoZnO). To check their size, micro and nanoZnO are submitted to a morphological study by FE-SEM (Fig. S3). MicroZnO micrograph (Fig. S3a) shows mainly hexagonal prismatic particles with lengths of 1-2 $\mu \mathrm{m}$. Regarding nanoZnO (Fig. S3b), the main morphology observed is disaggregated spherical particles with sizes of $15-30$ nm. As already mentioned, size and therefore the specific surface area are parameters that can influence the antimicrobial activity. Thus, specific surface area (SSA) of Ms-ZnO, microZnO and nanoZnO is measured (Fig. S4). As expected, the highest value corresponds with nanoZnO $\left(23.9 \mathrm{~m}^{2} \mathrm{~g}-1\right)$ followed by microZnO $\left(4.6 \mathrm{~m}^{2} \mathrm{~g}-1\right)$. Surprisingly, Ms-ZnO reaches the lowest SSA value $\left(1.6 \mathrm{~m}^{2} \mathrm{~g}^{-1}\right)$ in spite of their platelet microstructure.
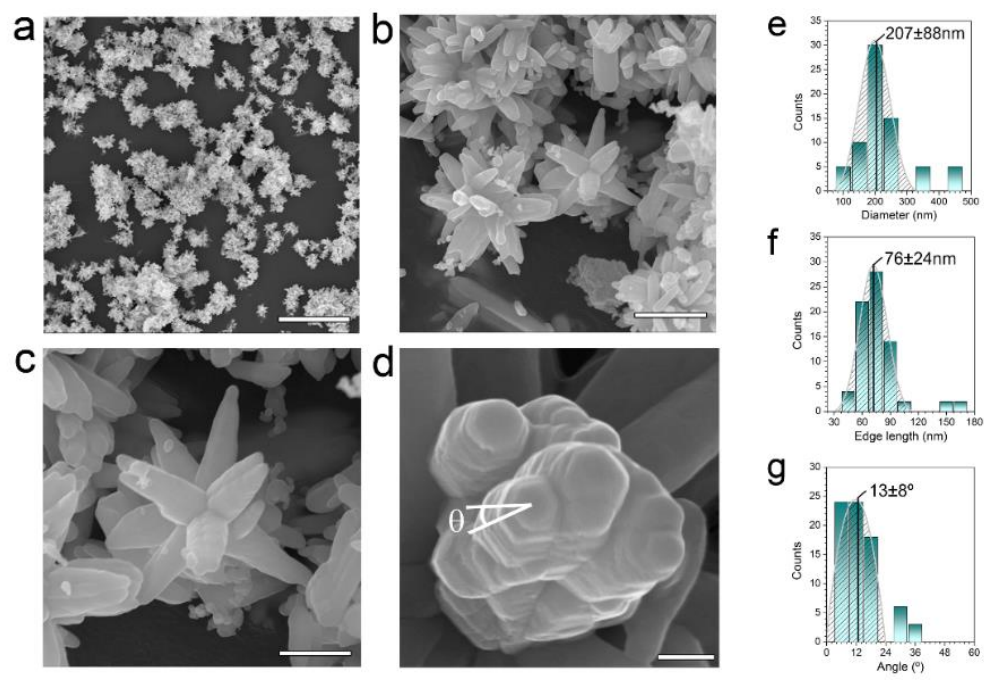
E. de Lucas-Gil, J.J. Reinosa, K. Neuhaus, L. Vera-Londono, M. Martín-González, J.F. Fernández, F. Rubio-Marcos, Exploring New Mechanisms for Effective Antimicrobial Materials: Electric Contact-Killing Based on Multiple Schottky Barriers, ACS Appl. Mater. Interfaces. 9 (2017) $26219-26225$. doi:10.1021/acsami.7b09695.

Figure 1 | Morphological characterization of Ms-ZnO. LR-SEM micrographs (a, b) show the main morphology in star-like structure of Ms-ZnO. HR-SEM micrographs (c, d) display the platelets organization of the Ms-ZnO structure. Scale bar of the panel a is $20 \mu \mathrm{m}$, panel b is $2 \mu \mathrm{m}$, panel $\mathrm{c}$ is $1 \mu \mathrm{m}$ and panel $\mathrm{d}$ is $200 \mathrm{~nm}$. Microstructural parameter characteristics of Ms-ZnO are platelet diameter (e), platelet thickness defined as edge length $(\mathbf{f})$ and rotation angle between platelets $(\mathrm{g})$. An example of rotation angle $(\theta)$ is represented in panel d.

Evaluation of antimicrobial activity. The antimicrobial capacity of $\mathrm{Ms}-\mathrm{ZnO}$ is tested for two kinds of microorganisms: bacteria and fungi (Fig. 2). Besides, the results obtained are compared with the microZnO and nanoZnO samples previously characterized (Fig. S3 and S4). The antibacterial activity is performed by the Macrodilution method from the National Committee of Clinical Laboratory Standards (NCCLS) with some modifications (see Experimental Section). The antibacterial test is realized against two bacteria: Escherichia coli (CECT 516) and Staphylococcus aureus (CECT 240) after the addition of 3 ppm of ZnO samples. The parameters selected to measure the antibacterial effectiveness are colony forming units (CFU) and antimicrobial activity value (denoted as R). For E. coli (Fig. 2a) the addition of microZnO and nanoZnO carries the same behavior. In both cases, the bacterial population decrease respect to initial population (called as control) until reaching acceptable R values around 2. Extraordinarily, the presence of Ms-ZnO inhibits bacterial growth to excellent $\mathrm{R}$ values greater than 3.6. The $S$. aureus test (Fig. $\mathbf{2 b}$ ) reveals a similar behavior than E. coli study. Again, the microZnO and nanoZnO $\mathrm{R}$ results are close to 2. Likewise, the $\mathrm{R}$ value of Ms-ZnO above 3 displays a better behavior than its competitors. For antifungal activity, a Kirby-Bauer method is performed (see Experimental Section). The fungus selected for this test is Aspergillus niger (CECT 2807) in spore form. The sporulation inhibition diameter (SID) of fungi provides the ZnO efficiency (Fig. S5). As shown in Fig. 2c, the highest SID value corresponds to $\mathrm{Ms}-\mathrm{ZnO}(35 \mathrm{~mm})$ while microZnO and nanoZnO SIDs are 24 and 27 mm, respectively. So once again, $\mathrm{Ms}-\mathrm{ZnO}$ shows the best performance with respect to two different $\mathrm{ZnO}$ size particles. To know how the addition of Ms-ZnO affects microorganisms, FE-SEM is performed. Before, microorganism morphologies are studied: E. coli (Fig. S6a) shows a rod-shaped morphology, S. aureus (Fig. S6b) is round-shaped and A. niger (Fig. S6c) are formed by globular spores. Then, colored micrographs obtained display interactions between Ms$\mathrm{ZnO}$ and E. coli (Fig. 2d), S. aureus (Fig. 2e) or A. niger (Fig. 2f), see original micrographs in Fig.S7. All micrographs show structural damage (pointed with arrows) such as wrinkled, flattened and/or cracked microorganism walls in comparison with initial morphologies. As a possible result, these disturbances in the envelope lead to membrane rupture and finally to the microorganism death. Highlight that in all cases are 
E. de Lucas-Gil, J.J. Reinosa, K. Neuhaus, L. Vera-Londono, M. Martín-González, J.F. Fernández, F. Rubio-Marcos, Exploring New Mechanisms for Effective Antimicrobial Materials: Electric Contact-Killing Based on Multiple Schottky Barriers, ACS Appl. Mater. Interfaces. 9 (2017) $26219-26225$. doi:10.1021/acsami.7b09695.

necessary the direct contact between $\mathrm{Ms}-\mathrm{ZnO}$ and microorganism to cause injuries. These results show a robust antimicrobial activity of $\mathrm{Ms}-\mathrm{ZnO}$, both bacteria and fungi, in spite of its size and low specific surface area.

a
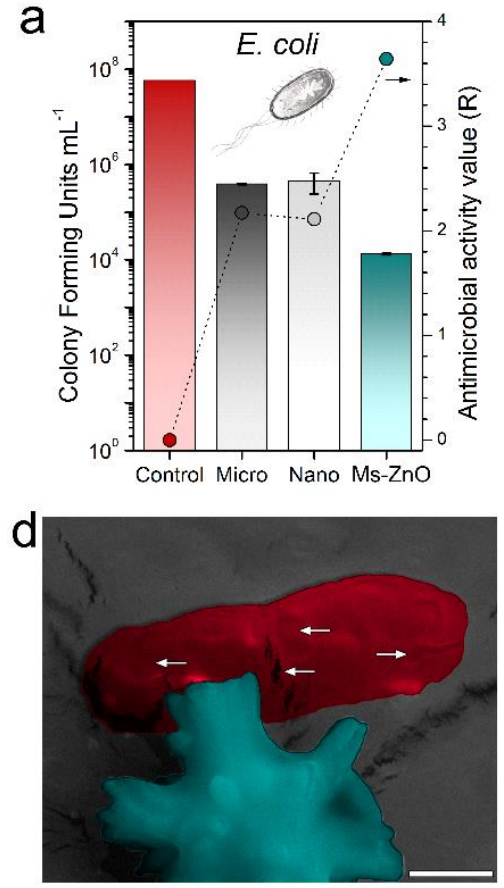

b
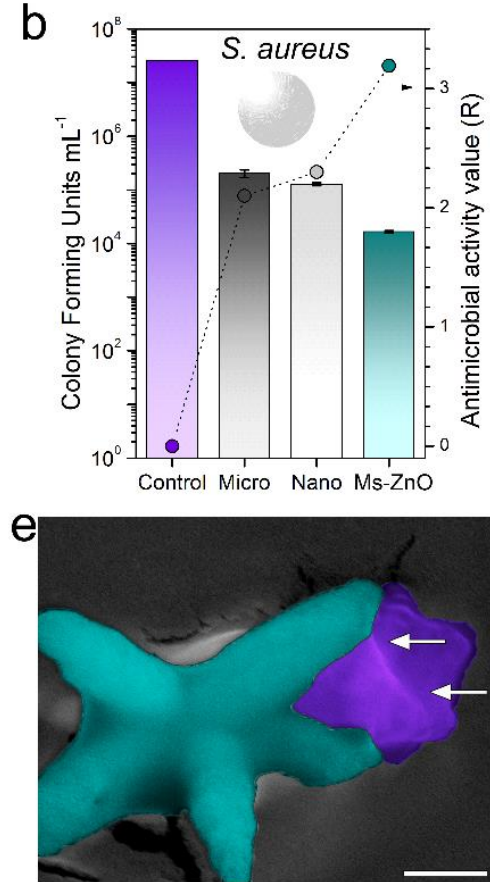
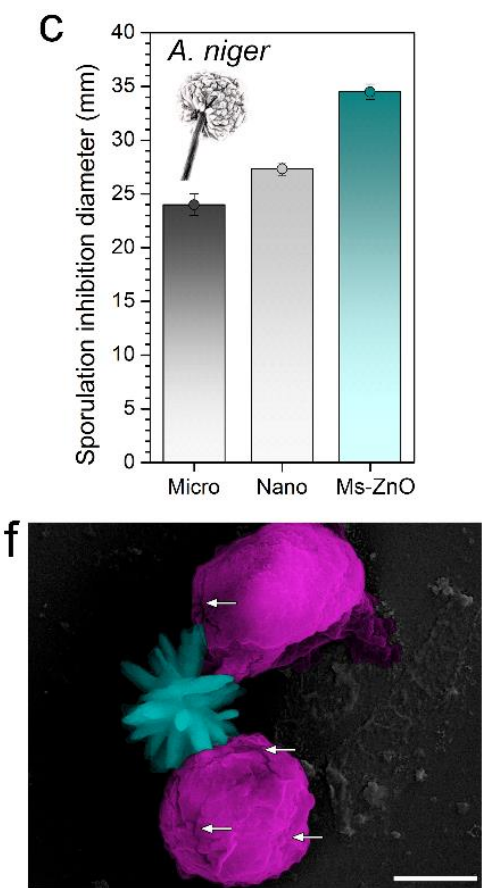

Figure 2 | Antimicrobial activity of star-like Ms-ZnO. Panel a and $\mathbf{b}$ shows the antibacterial activity against E. coli and S. aureus, respectively. The antibacterial activity is expressed as colony forming units per $\mathrm{mL}$ (CFU mL-1) and as antimicrobial activity value (R). Panel c displays the antifungal activity against $A$. niger expressed as sporulation inhibition diameter (SID). The initial bacteria count (control) is represented by red (E. coli) or purple (S. aureus), two size references (micro and nanoZnO) are denoted by grey and Ms-ZnO is symbolized by blue. Each column represents an average of triplicate measurements for 3 ppm concentration of $\mathrm{ZnO}$ in the case of bacteria (E. coli and S. aureus) and for $0.01 \%$ of $\mathrm{ZnO}$ suspension in case of fungi (A. niger). CFU and SID data are shown with mean values and standard error bars. FE-SEM micrographs illustrate the E. coli (d), S. aureus (e) and A. niger (f) after the Ms-ZnO incorporation. Coloured micrographs have been selected to facilitate the identification of microorganisms and particles (see original micrographs in Fig. S7). White arrows indicate some damage caused by Ms-ZnO. Scale bar of panel dis $1 \mu \mathrm{m}$, panel e is $500 \mathrm{~nm}$ and panel $\mathrm{f}$ is $2 \mu \mathrm{m}$.

Knowing the action mechanisms against microorganism. Once the microorganism damages are known, it is important to analyse why they occur. As discussed above, the main mechanisms can be classified into two types: chemical or physical. To know the relevance of chemical interactions in Ms- $\mathrm{ZnO}$, a leaching assay of $\mathrm{Zn}^{2+}$ is performed by using ICP-OES. Fig. 3a shows the $\mathrm{Zn}^{2+}$ released for Ms-ZnO compared with nanoZnO and microZnO. The sample that releases more $\mathrm{Zn}^{2+}$ cation to the medium is nanoZnO $\left(6.44 \mathrm{mg} \mathrm{L}^{-1}\right)$. On the other hand, microZnO (0.59 $\left.\mathrm{mg} \mathrm{L}^{-1}\right)$ and $\mathrm{Ms}-\mathrm{ZnO}\left(0.49 \mathrm{mg} \mathrm{L}^{-1}\right)$ display very low $\mathrm{Zn}^{2+}$ released below $1 \mathrm{mg} \mathrm{L}^{-1}$. This implies that size and specific surface area play an important role in leaching. The low $\mathrm{Zn}^{2+}$ leaching of Ms- $\mathrm{ZnO}$ is due to cations retention in the structure and therefore, the non-release into the environment. Equally important, within the chemical mechanism of $\mathrm{Ms}-\mathrm{ZnO}$, is to know the role of reactive oxygen species (ROS). To 
E. de Lucas-Gil, J.J. Reinosa, K. Neuhaus, L. Vera-Londono, M. Martín-González, J.F. Fernández, F. Rubio-Marcos, Exploring New Mechanisms for Effective Antimicrobial Materials: Electric Contact-Killing Based on Multiple Schottky Barriers, ACS Appl. Mater. Interfaces. 9 (2017) $26219-26225$. doi:10.1021/acsami.7b09695.

measure ROS generation, a photocatalytic study is performed. During a photocatalysis process, ROS generated plays as active species in the oxidation of dyes, especially hydroxyl radicals $\left(\mathrm{OH}^{\circ}\right)$ and superoxide anion radicals $\left(\mathrm{O}_{2}^{-\cdot}\right)$. The photocatalytic test is carried out by photodegradation of a standard organic dye, methyl orange (MO), under UV light irradiation. To calculate the degradation percentage, Beer-Lambert law is used. Fig. $\mathbf{3 b}$ displays the degradation of $\mathrm{Ms}-\mathrm{ZnO}$ compared with microZnO and nanoZnO. The reference structures, microZnO and nanoZnO show excellent photocatalytic behaviour, practically degrading $100 \%$ of the dye to $2 \mathrm{~h}$ and $3 \mathrm{~h}$, respectively. However, Ms-ZnO cannot degrade methyl orange dye to $30 \%$ after $5 \mathrm{~h}$. Therefore, relating photocatalytic activity to ROS generation, it can be said that the hierarchical Ms-ZnO structure generates about $72 \%$ less ROS activity than micrometric or nanometric structures. According to these results, the chemical interaction importance is not relevant to the $\mathrm{Ms}-\mathrm{ZnO}$ antimicrobial mechanism.

a
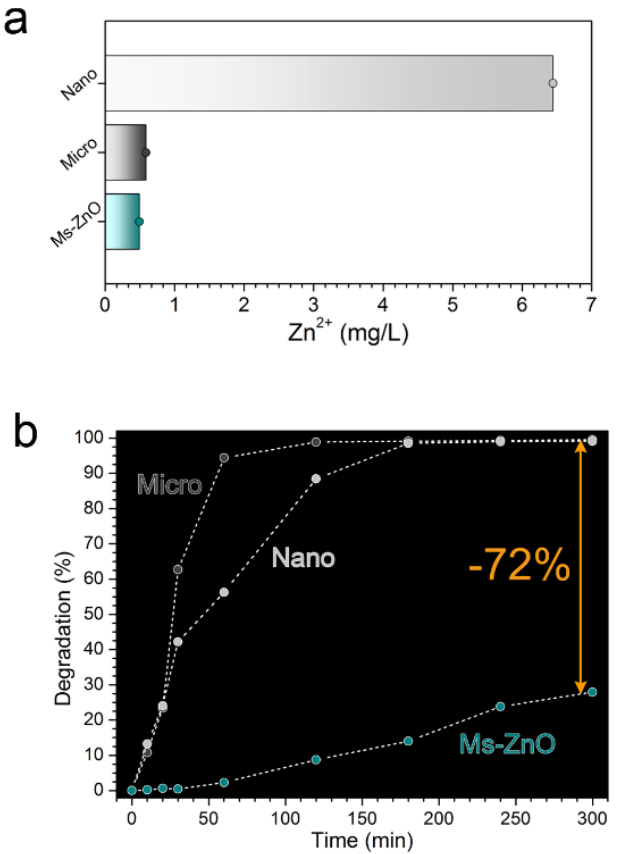

Figure 3 | Relevance studies of chemical interactions in antimicrobial activity of Ms-ZnO. Leaching essays of $\mathrm{Zn}^{2+}$ in peptone water (a) and photocatalytic degradation of methyl orange (MO) exposure to UV light irradiation (b) of commercial references microZnO (dark grey) and nanoZnO (light grey) and $\mathrm{Ms}-\mathrm{ZnO}$ (blue).

About physical interactions, it is important to know the physical characteristics of Ms-ZnO. The surface charge of Ms-ZnO is performed by zeta potential. As shown in Fig. 4a, Ms-ZnO has a negative surface potential (about $-20 \mathrm{mV}$ ) between the $\mathrm{pH}$ ranges of 6 to 10 . Hence, Ms- $\mathrm{ZnO}$ has a stable negative potential in a $\mathrm{pH}$ range where microorganisms proliferate, close to neutral $\mathrm{pH}$. As indicated by the conductivity, out of that range begins the 
E. de Lucas-Gil, J.J. Reinosa, K. Neuhaus, L. Vera-Londono, M. Martín-González, J.F. Fernández, F. Rubio-Marcos, Exploring New Mechanisms for Effective Antimicrobial Materials: Electric Contact-Killing Based on Multiple Schottky Barriers, ACS Appl. Mater. Interfaces. 9 (2017) $26219-26225$. doi:10.1021/acsami.7b09695.

solution of $\mathrm{Ms}-\mathrm{ZnO}$ to the medium. Cathodoluminescence (CL) provides data on ZnO structure defects. Panchromatic map of Ms-ZnO (Fig. 4b) displays that most intensity emissions come from the top of platelet stacks. Moreover, the stacked platelets grow vertically along c-axis, so the emissions most probably are due to polar (0001) surface. The CL emission spectra (Fig. S8) exhibit two emissions: the near-band edge (NBE) at 385 $\mathrm{nm}$ and green luminescence (GL) band at $590 \mathrm{~nm}$. The GL/NBE intensity ratio is commonly considered as a benchmark of the crystalline quality (point defects concentration). ${ }^{23}$ The integrated intensity GL/NBE ratio of horizontal branch (1) is 0.89 while GL/NBE ratio of vertical branch (2) is 2.43. Therefore, most luminescent areas, which are mostly terminal zones, correspond to defects in the $\mathrm{ZnO}$ crystal. Fig. 4c shows Ms-ZnO against A. niger. Again, it is possible to observe that branch tips have more intensity (that is, in those areas a higher defect number in $\mathrm{ZnO}$ crystal are concentrated).
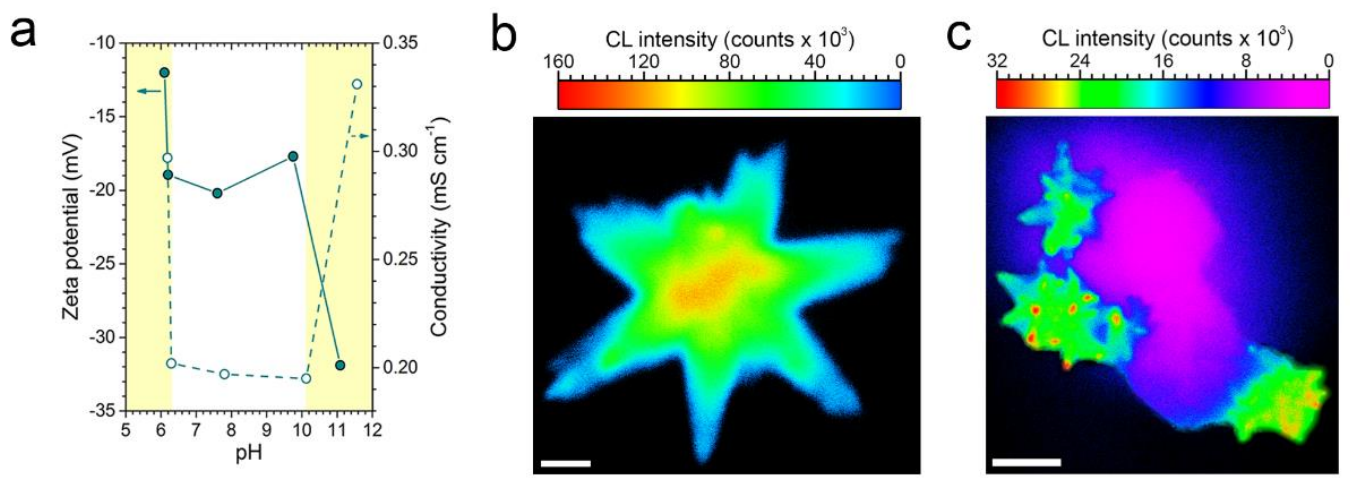

Figure 4 | Relevance studies of physical interactions in antimicrobial activity of Ms-ZnO. Zeta potential (a), SEM-CL panchromatic of $\mathrm{Ms}-\mathrm{ZnO}(\mathbf{b})$ and its effect against fungi (c). Scale bar of panel $\mathrm{d}$ is $500 \mathrm{~nm}$ and panel e is $2 \mu \mathrm{m}$.

In addition, Kelvin Probe Force Microscopy (KPFM) is obtained to complement the CL measurements. As shown in the topography (Fig. 5a) and the surface potential (Fig. 5b), a high defect concentration is found at the branch tips of Ms-ZnO. Observing in more detail, important changes in the surface potential difference $\Delta \Phi_{\mathrm{SP}}$ are observed between bulk and the branch tips, in the range of roughly 30-50 mV. After a meticulous analysis of a section (Fig. 5c), we also find a direct relation between the potential difference and the morphology height, one increases as the other decreases (highlighted in yellow shading). 
E. de Lucas-Gil, J.J. Reinosa, K. Neuhaus, L. Vera-Londono, M. Martín-González, J.F. Fernández, F. Rubio-Marcos, Exploring New Mechanisms for Effective Antimicrobial Materials: Electric Contact-Killing Based on Multiple Schottky Barriers, ACS Appl. Mater. Interfaces. 9 (2017) $26219-26225$. doi:10.1021/acsami.7b09695.
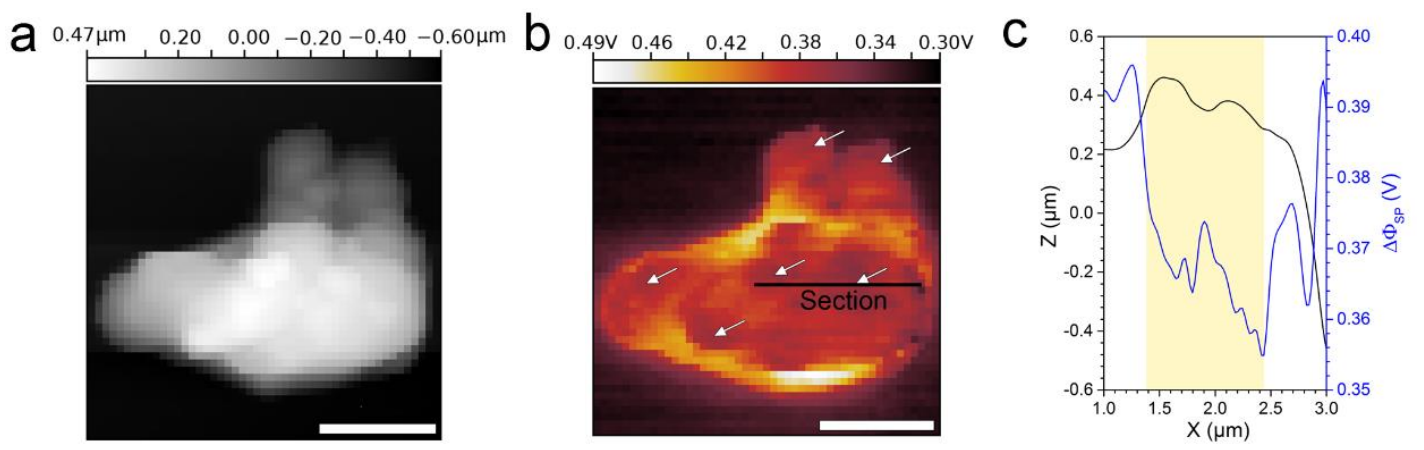

Figure 5 | Study of charge accumulation on Ms-ZnO surface. KPFM topography (a), surface potential (b) and section graphic of surface potential (c). The most important changes of potential are indicated with white arrows. The graph $\mathrm{h}$ section is indicated in panel $\mathrm{g}$ with a black line. Scale bar is $1 \mu \mathrm{m}$.

Charge accumulation in the structure, why? As determined by $\mathrm{XRD}, \mathrm{Ms}-\mathrm{ZnO}$ crystallizes mainly in the most stable hexagonal wurtzite structure (Fig. S1) preferably growing along the c-axis. This structure is constituted by a polar crystal which is characterized by a positively charged Zn-polar face and a negatively charged O-polar face in addition to the non-polar faces (Fig. 6a). ${ }^{24}$ The surface polarity of structure is associated with Zn and O atoms placed in terminal positions. Transferred this concept to platelets, the hexagonal prism is built by a positive or negative charged base, depending on whether the terminal atoms are zinc or oxygen respectively, and six non-charged sides (Fig $\mathbf{6 b}$ ). Possibly, these positively and negatively charged surfaces are involved in the mechanism behind the formation of stacked platelets. The electrostatic attraction between these polar surfaces makes them join with each other, allowing the platelets stacking along c-axis (Fig. 6c). ${ }^{25}$ A particularity of Ms-ZnO is the platelets rotation, which makes a non-perfect crystallographic plane alignment. The polarity, the stacking along c-axis and the platelets rotation may effect on Ms-ZnO properties, as it happens with defects in crystals. A domain wall is created between platelets. Extraordinarily, this morphology allows producing multiple domains wall in each $\mathrm{ZnO}$ microparticle. Given the possible size differences and rotated orientation of platelets, the conduction bands of n-type semiconductor $\mathrm{Ms}-\mathrm{ZnO}$ are altered. These results lead to the generation of potential energy barriers, known as a Schottky barrier $\left(\Phi_{\mathrm{SB}}\right)$, see Fig. 6d. For electronic transport, the Schottky barrier modifies the flow of electrons through the Ms-ZnO structure allowing a negative charge accumulation at these barriers. The stacked morphology facilitates the negative electric charge accumulation throughout the structure as shown in zeta potential (Fig. 4a). In addition, the surface potential changes observed in KPFM images (Fig. 5) indicate local negative charge accumulation. ${ }^{26}$ This behavior resembles a charged domain wall (CDW) where uncompensated charge is accumulated at the domain walls (DWs). ${ }^{27,28} \mathrm{~A}$ domain 
E. de Lucas-Gil, J.J. Reinosa, K. Neuhaus, L. Vera-Londono, M. Martín-González, J.F. Fernández, F. Rubio-Marcos, Exploring New Mechanisms for Effective Antimicrobial Materials: Electric Contact-Killing Based on Multiple Schottky Barriers, ACS Appl. Mater. Interfaces. 9 (2017) $26219-26225$. doi:10.1021/acsami.7b09695.

wall (DW) is an interface separating crystallographic domains. So, extrapolating these terms to Ms-ZnO, we get a new domain structure. The new definition of "domain" is a region within a ZnO crystal in which the crystal is homogeneous in a crystallographic direction. In this case, each platelet is a single domain, and it is the boundaries formed by each platelet what gives rise to finite interfacial phenomena and the appearance of domain walls. Thus, the presence of multiple stacked platelets gives rise to the appearance of multi-domains. In this context, "domain walls" are archetypal homointerfaces between platelets and refer to interplatelet layers. The "domain walls" lead to a generation of Schottky barriers, where a negative charge is accumulated at the different conduction band energies of platelets. The Schottky barriers generation is an atypical case of $\mathrm{ZnO}$ microparticles and exclusive of ceramics and thin film heteroestructures. However, the appearance of Schottky barriers in $\mathrm{Ms}-\mathrm{ZnO}$ are originated at the interfaces by the rotated platelets. Finally, the charge accumulation at "domain walls" generates an electric potential or "charged domain walls". The more defects or crystallographic imperfections, the more charge accumulation.

a

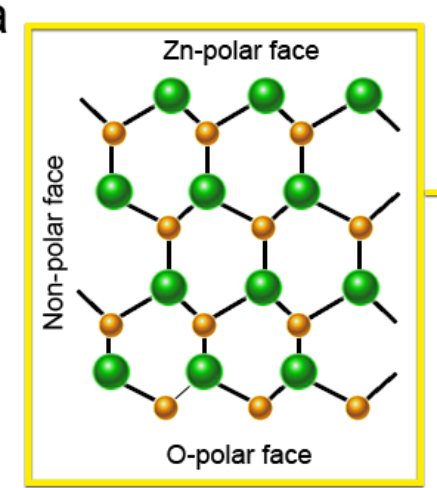

C

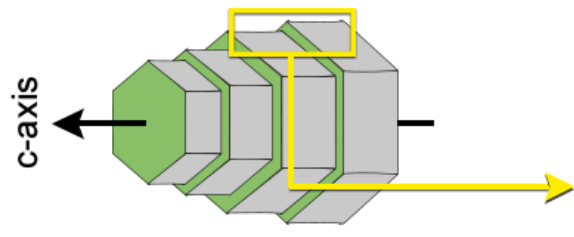

b

d

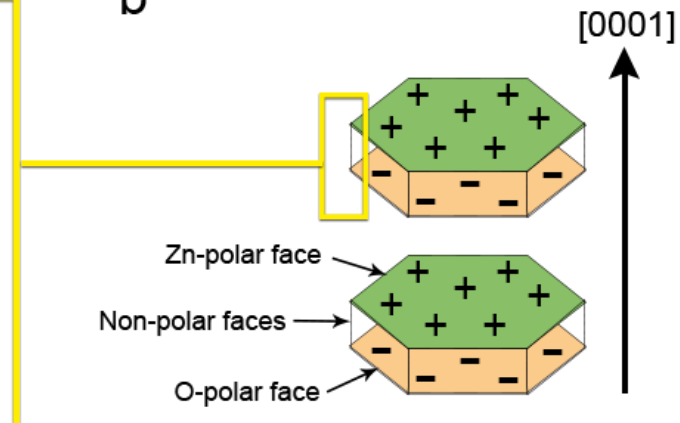

Interplatelet layer

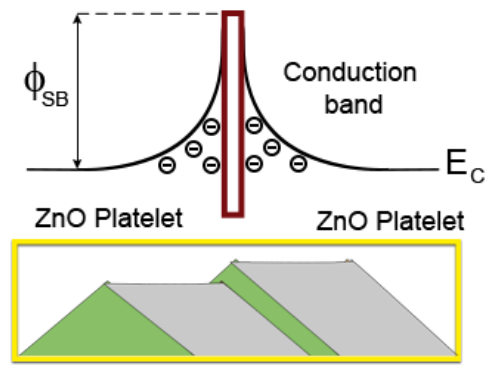

Figure 6 | Platelet structure and generation of Schottky barriers. Hexagonal wurtzite structure (a) built by positive Zn-polar, negative Opolar or non-polar faces. Panel $\mathbf{b}$ shows two platelets of Ms-ZnO stacked along [0001] direction. The platelet of Ms-ZnO stacking along caxis (c) generates a potential barrier called Schottky barrier $\left(\Phi_{\mathrm{SB}}\right)$ at interplatelet layers where negative charge is accumulated (d).

This hypothesis on the accumulation of charge in the domain walls can be confirmed with KPFM measurements. A branch of $\mathrm{Ms}-\mathrm{ZnO}$ structure is selected to perform a more exhaustive analysis of KPFM. The branch 
E. de Lucas-Gil, J.J. Reinosa, K. Neuhaus, L. Vera-Londono, M. Martín-González, J.F. Fernández, F. Rubio-Marcos, Exploring New Mechanisms for Effective Antimicrobial Materials: Electric Contact-Killing Based on Multiple Schottky Barriers, ACS Appl. Mater. Interfaces. 9 (2017) $26219-26225$. doi:10.1021/acsami.7b09695.

topography (Fig. 7a) displays the rotated platelets, creating a disordered stack. As revealed in Fig. $7 \mathbf{b}$, there is a negative contact potential difference (CPD) between the tip and the platelets. Moreover, the relative CPD values are more pronounce at the edge regions which agrees with uncompensated charge at the Schottky barrier (Fig. 7c). Thus, when Ms-ZnO has direct contact with microorganisms, the electric potential produced by higher charged areas directly affects them. This behavior resembles the technique known as electroporation, where cell membranes are exposed to high pulsed electric fields inducing cell apoptosis. ${ }^{29,30}$
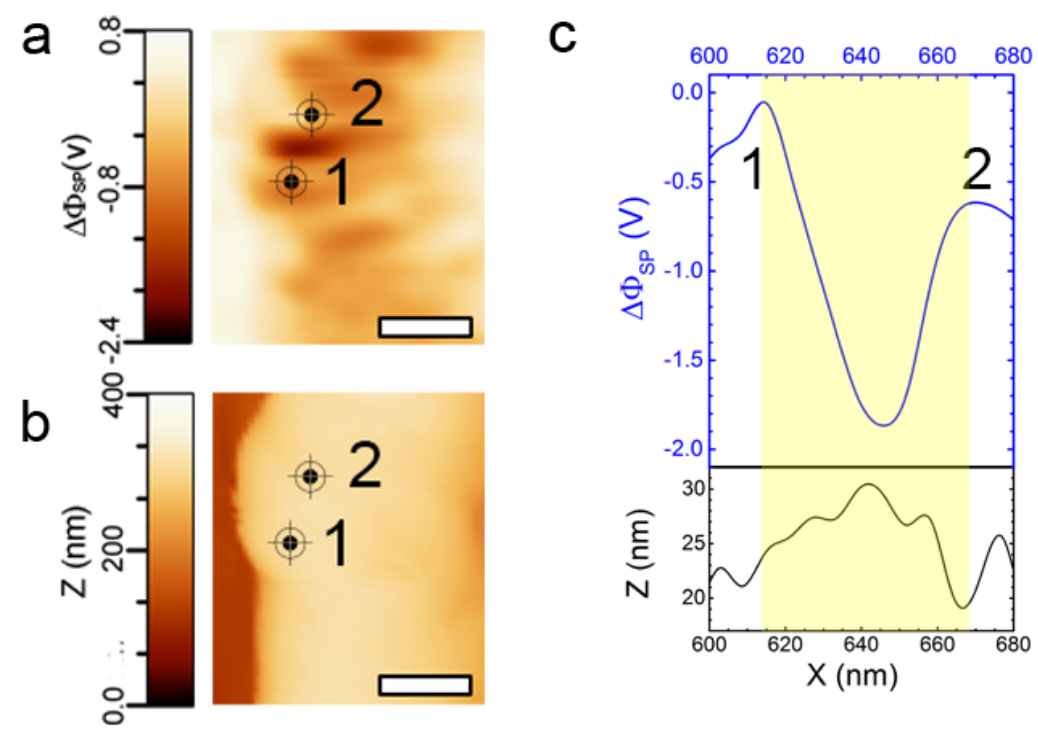

Figure 7 | Evidence of Schottky barriers appearance in Ms-ZnO. Detailed surface potential (a) and topography of a rotated platelet stack (b) that shows charge accumulation in Schottky barriers evidenced by KPFM (c). Scale bar is $100 \mathrm{~nm}$.

A discharge test is realized to determine the maximum discharge potential of a Ms-ZnO particle (Fig. 8). The topography image (Fig. 8a) shows the particle morphology. Analyzing the relative height (Fig. 8b), two zones are selected: one on the top and another to the bottom of particle. The Ms-ZnO particle placed on a metal surface in open circuit mode is capable of being charged. When the charged particle comes into contact with AFM tip, an electric shock occurs. The results of this discharge is shown in the surface potential image, Fig. 8c. Taking as reference the same top and bottom zones, the contact potential difference (Fig. $8 \mathbf{d})$ reaches values up to $-3.5 \mathrm{~V}$ for the higher zones. This potential value $(-3.5 \mathrm{~V})$ corresponds to the charge collected from two or three adjacent domain pairs, between -1.5 to $-2 \mathrm{~V}$ (see Fig.7c), to the discharge point. Therefore, Ms-ZnO acts as a pulsed electric field with a discharge potential against the cell membrane. The Ms-ZnO high electric field created, which exceeds the breakdown potential of the lipid bilayer, ${ }^{31}$ causes irreparable damage to the membrane finally 
E. de Lucas-Gil, J.J. Reinosa, K. Neuhaus, L. Vera-Londono, M. Martín-González, J.F. Fernández, F. Rubio-Marcos, Exploring New Mechanisms for Effective Antimicrobial Materials: Electric Contact-Killing Based on Multiple Schottky Barriers, ACS Appl. Mater. Interfaces. 9 (2017) $26219-26225$. doi:10.1021/acsami.7b09695.

causing its rupture. As the generated charges are a consequence of electron accumulation in Schottky barriers, the Ms-ZnO does not require external stimulation like photostimulation. Moreover, the large number of Schottky barriers through the Ms-ZnO structure allows maintaining their activity for long periods of time. Consequently, the Ms-ZnO is able to keep long-lasting activity and very low concentrations or dose with excellent results.
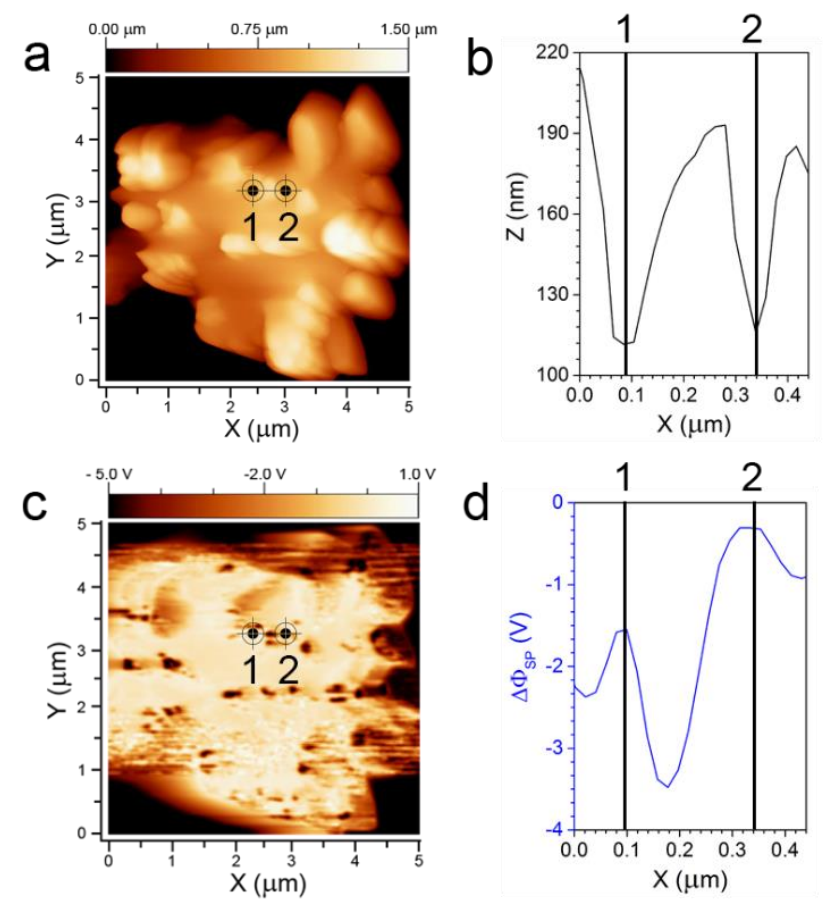

Figure 8 | Discharge potential of Ms-ZnO particle. Topography image (a) and relative height graphic (b) of Ms-ZnO particle. Surface potential image (c) and contact potential difference graphic (d) of the same particle. The particle top area is represented by number 1 and the particle bottom corresponds to number 2 .

\section{Conclusions}

In summary, we are able to create a microstructured $\mathrm{ZnO}, \mathrm{Ms}-\mathrm{ZnO}$, with excellent antimicrobial properties, both bacteria and fungi. To discover the origin of $\mathrm{Ms}-\mathrm{ZnO}$ outstanding antimicrobial activity, different action mechanisms have been studied. The most common mechanisms are divided into chemicals and physical. Evaluating the main chemical interactions, $\mathrm{Zn}^{2+}$ released and ROS generation, we have observed that they do not play a relevant role. However, we were struck by the negative zeta potential discovery of Ms-ZnO. This opens the possibility of the performance of the physical mechanisms. The cathodoluminiscence indicated us the zones with highest crystal defect concentration. The negative charge accumulation is confirmed by KPFM 
E. de Lucas-Gil, J.J. Reinosa, K. Neuhaus, L. Vera-Londono, M. Martín-González, J.F. Fernández, F. Rubio-Marcos, Exploring New Mechanisms for Effective Antimicrobial Materials: Electric Contact-Killing Based on Multiple Schottky Barriers, ACS Appl. Mater. Interfaces. 9 (2017) $26219-26225$. doi:10.1021/acsami.7b09695

measurements, i.e. the negative electric potential of Ms-ZnO. Moreover, KPFM indicates that the accumulation occurs mainly in the edge platelets areas with the highest defect number observed in CL. The explanation of this peculiar property is based on the platelet stacked structure of Ms-ZnO. Each platelet junction forms a domain wall due to the differences between the conduction bands of the platelets. The electron flow is retained in these areas generating a charged domain wall or potential energy barrier, known as a Schottky barrier ( $\left.\Phi_{\mathrm{SB}}\right)$. Importantly, our results demonstrate that stepping up the physical interaction role of the antimicrobial agent through an effective direct contact with microorganisms by electric discharge, also results in a long-lasting antimicrobial property. These findings point the way towards more stable antimicrobial agent designs to combat multidrug-resistant microbial in the field of biomedical and environmental applications.

\section{References}

(1) Tacconelli, E.; Magrini, N.; Kahlmeter, G.; Singh, N. Global Priority List Of Antibiotic-Resistant Bacteria To Guide Research, Discovery, And Development Of New Antibiotics. World Health Organization. 2017, pp 1-7.

(2) Moellering, R. C. NDM-1 - A Cause for Worldwide Concern. N. Engl. J. Med. 2010, 363 (25), 2377-2379.

(3) Bush, K.; Jacoby, G. A. Updated Functional Classification of Beta-Lactamases. Antimicrob. Agents Chemother. 2010, 54 (3), $969-$ 976.

(4) Chua, S. L.; Yam, J. K. H.; Hao, P.; Adav, S. S.; Salido, M. M.; Liu, Y.; Givskov, M.; Sze, S. K.; Tolker-Nielsen, T.; Yang, L. Selective Labelling and Eradication of Antibiotic-Tolerant Bacterial Populations in Pseudomonas Aeruginosa Biofilms. Nat. Commun. 2016, 7, 10750.

(5) Hoop, M.; Shen, Y.; Chen, X.-Z.; Mushtaq, F.; Iuliano, L. M.; Sakar, M. S.; Petruska, A.; Loessner, M. J.; Nelson, B. J.; Pané, S. Magnetically Driven Silver-Coated Nanocoils for Efficient Bacterial Contact Killing. Adv. Funct. Mater. 2015, 1-7.

(6) Hasan, J.; Chatterjee, K. Recent Advances in Engineering Topography Mediated Antibacterial Surfaces. Nanoscale 2015, 7 (38), $15568-15575$.

(7) Ann, L. C.; Mahmud, S.; Bakhori, S. K. M.; Sirelkhatim, A.; Mohamad, D.; Hasan, H.; Seeni, A.; Rahman, R. A. Antibacterial Responses of Zinc Oxide Structures against Staphylococcus Aureus, Pseudomonas Aeruginosa and Streptococcus Pyogenes. Ceram. Int. 2014, 40 (2), 2993-3001.

(8) Sawai, J. Quantitative Evaluation of Antibacterial Activities of Metallic Oxide Powders ( $\mathrm{ZnO}, \mathrm{MgO}$ and CaO) by Conductimetric Assay. J. Microbiol. Methods 2003, 54 (2), 177-182.

(9) Jones, N.; Ray, B.; Ranjit, K. T.; Manna, A. C. Antibacterial Activity of ZnO Nanoparticle Suspensions on a Broad Spectrum of Microorganisms. FEMS Microbiol. Lett. 2008, 279 (1), 71-76.

(10) Li, M.; Zhu, L.; Lin, D. Toxicity of ZnO Nanoparticles to Escherichia Coli: Mechanism and the Influence of Medium Components. Environ. Sci. Technol. 2011, 45 (5), 1977-1983.

(11) Ohira, T.; Yamamoto, O.; lida, Y.; Nakagawa, Z.-E. Antibacterial Activity of ZnO Powder with Crystallographic Orientation. J. Mater. Sci. Mater. Med. 2008, 19 (3), 1407-1412.

(12) Sharma, D.; Rajput, J.; Kaith, B. S.; Kaur, M.; Sharma, S. Synthesis of ZnO Nanoparticles and Study of Their Antibacterial and Antifungal Properties. Thin Solid Films 2010, 519 (3), 1224-1229.

(13) Lipovsky, A.; Nitzan, Y.; Gedanken, A.; Lubart, R. Antifungal Activity of ZnO Nanoparticles-the Role of ROS Mediated Cell Injury. Nanotechnology 2011, 22 (10), 105101-1-5.

(14) Zhang, L.; Jiang, Y.; Ding, Y.; Daskalakis, N.; Jeuken, L.; Povey, M.; O’Neill, A. J.; York, D. W. Mechanistic Investigation into Antibacterial Behaviour of Suspensions of ZnO Nanoparticles against E. Coli. J. Nanoparticle Res. 2010, 12 (5), $1625-1636$.

(15) Applerot, G.; Lipovsky, A.; Dror, R.; Perkas, N.; Nitzan, Y.; Lubart, R.; Gedanken, A. Enhanced Antibacterial Activity of Nanocrystalline ZnO due to Increased ROS-Mediated Cell Injury. Adv. Funct. Mater. 2009, 19 (6), 842-852.

(16) Li, Y.; Zhang, W.; Niu, J.; Chen, Y. Mechanism of Photogenerated Reactive Oxygen Species and Correlation with the Antibacterial Properties of Engineered Metal-Oxide Nanoparticles. ACS Nano 2012, 6 (6), 5164-5173.

(17) Sirelkhatim, A.; Mahmud, S.; Seeni, A.; Kaus, N. H. M.; Ann, L. C.; Bakhori, S. K. M.; Hasan, H.; Mohamad, D. Review on Zinc Oxide Nanoparticles: Antibacterial Activity and Toxicity Mechanism. Nano-Micro Lett. 2015, 7 (3), $219-242$.

(18) Pasquet, J.; Chevalier, Y.; Pelletier, J.; Couval, E.; Bouvier, D.; Bolzinger, M.-A. The Contribution of Zinc lons to the Antimicrobial Activity of Zinc Oxide. Colloids Surf., A 2014, 457, 263-274.

(19) Nel, A. E.; Mädler, L.; Velegol, D.; Xia, T.; Hoek, E. M. V; Somasundaran, P.; Klaessig, F.; Castranova, V.; Thompson, M. 
E. de Lucas-Gil, J.J. Reinosa, K. Neuhaus, L. Vera-Londono, M. Martín-González, J.F. Fernández, F. Rubio-Marcos, Exploring New Mechanisms for Effective Antimicrobial Materials: Electric Contact-Killing Based on Multiple Schottky Barriers, ACS Appl. Mater. Interfaces. 9 (2017) 26219-26225. doi:10.1021/acsami.7b09695.

(20) Espitia, P. J. P.; Soares, N. de F. F.; Coimbra, J. S. dos R.; de Andrade, N. J.; Cruz, R. S.; Medeiros, E. A. A. Zinc Oxide Nanoparticles: Synthesis, Antimicrobial Activity and Food Packaging Applications. Food Bioprocess Technol. 2012, 5 (5), 1447-1464.

(21) Padmavathy, N.; Vijayaraghavan, R. Enhanced Bioactivity of ZnO Nanoparticles-an Antimicrobial Study. Sci. Technol. Adv. Mater. 2008, 9 (3), 35004.

(22) Jiang, Y.; Zhang, L.; Wen, D.; Ding, Y. Role of Physical and Chemical Interactions in the Antibacterial Behavior of ZnO Nanoparticles against E. Coli. Mater. Sci. Eng. C 2016, 69, 1361-1366.

(23) Fabbri, F.; Villani, M.; Catellani, a; Calzolari, a; Cicero, G.; Calestani, D.; Calestani, G.; Zappettini, a; Dierre, B.; Sekiguchi, T.; Salviati, G. Zn Vacancy Induced Green Luminescence on Non-Polar Surfaces in ZnO Nanostructures. Sci. Rep. 2014, 4, 5158.

(24) Ching, K.-L.; Li, G.; Ho, Y.-L.; Kwok, H.-S. The Role of Polarity and Surface Energy in the Growth Mechanism of ZnO from Nanorods to Nanotubes. CrystEngComm 2016, 18 (5), 779-786.

(25) Wang, S. S.; Xu, A. W. Template-Free Facile Solution Synthesis and Optical Properties of ZnO Mesocrystals. CrystEngComm 2013, 15 (2), 376-381.

(26) Bayerl, D. J.; Wang, X. Three-Dimensional Kelvin Probe Microscopy for Characterizing in-Plane Piezoelectric Potential of Laterally Deflected ZnO Micro-/nanowires. Adv. Funct. Mater. 2012, 22 (3), 652-660.

(27) Rubio-Marcos, F.; Del Campo, A.; Marchet, P.; Fernández, J. F. Ferroelectric Domain Wall Motion Induced by Polarized Light. Nat. Commun. 2015, 6, 6594.

(28) Jia, C.-L.; Mi, S.-B.; Urban, K.; Vrejoiu, I.; Alexe, M.; Hesse, D. Atomic-Scale Study of Electric Dipoles near Charged and Uncharged Domain Walls in Ferroelectric Films. Nat. Mater. 2008, 7 (1), 57-61.

(29) Chen, C.; Smye, S. W.; Robinson, M. P.; Evans, J. A. Membrane Electroporation Theories: A Review. Med. Biol. Eng. Comput. 2006, 44 (1-2), 5-14.

(30) Saulis, G. Electroporation of Cell Membranes: The Fundamental Effects of Pulsed Electric Fields in Food Processing. Food Eng. Rev. 2010, 2 (2), 52-73.

(31) Unal, R.; Yousef, A. E.; Dunne, C. P. Spectrofluorimetric Assessment of Bacterial Cell Membrane Damage by Pulsed Electric Field. Innovative Food Sci. Emerging Technol. 2002, 3 (3), 247-254.

\section{Associated content}

\section{Supporting Information}

The Supporting Information is available free of charge on the ACS Publications website.

Experimental details, Structural characterization of Ms-ZnO, Morphological characterization of different size $\mathrm{ZnO}$, Determination of specific surface area (SSA), Sporulation inhibition diameter (SID) to determine antifungal activity, Morphological characterization of microorganisms, Cathodoluminiscence emission spectra of Ms- $\mathrm{ZnO}$ in two points, Maximum discharge potential of Ms-ZnO particle.

\section{Acknowledgements}

The authors express their thanks to the MINECO (Spain) project MAT2013-48009-C4-1-P for their financial support. The Spanish National Research Council (CSIC) under project NANOMIND CSIC 201560E068. F. R-M is also indebted to MINECO for a 'Ramon y Cajal' contract (ref: RyC-2015-18626), which is co-financed by the European Social Fund. Finally, authors are grateful to Mercedes Monte-Serrano, Santiago De-Bernardi and Javier Menéndez for antimicrobial activity test, to $\mathrm{M}^{\mathrm{a}}$ José Velasco and Pilar Ortega for ICP-OES measurements and to Dr. Laura Tormo and Marta Furió of Museo Nacional of Ciencias Naturales (Madrid, Spain) for SEM-CL measurements.

Competing financial interests: The authors declare no competing financial interests. 